\title{
Platelet-mediated adhesion facilitates leukocyte sequestration in hypoxia-reoxygenated microvessels
}

\author{
Senfeng Zheng ${ }^{1}$, Yanting Cao ${ }^{1}$, Wenjian Zhang ${ }^{2}$, Honglin Liu ${ }^{2}$, Jia You $^{2}$, Yiqing Yin ${ }^{1}$, \\ Jinning Lou $^{2} \&$ Chenghui $\mathrm{Li}^{1 *}$ \\ ${ }^{1}$ Department of Anesthesiology, China-Japan Friendship Hospital, Beijing 100029, China; \\ ${ }^{2}$ Institute of Clinical Medical Science, China-Japan Friendship Hospital, Beijing 100029, China
}

Received August 30, 2015; accepted September 24, 2015; published online January 15, 2016

\begin{abstract}
Leukocyte transendothelial migration and sequestration are two distinct outcomes following leukocyte adhesion to endothelium during ischemia-reperfusion injury, in which platelets may play a pivotal role. In the present study, we established an in vitro hypoxia-reoxygenation model to mimic ischemia-reperfusion injury and found platelet pre-incubation significantly increased leukocyte adhesion to endothelial cells after hyoxia-reoxygenation (over 67\%). Blockade of endothelial-cell-expressed adhesion molecules inhibited leukocyte direct adhesion to endothelial cells, while platelet-mediated leukocyte adhesion was suppressed by blockade of platelet-expressed adhesion molecules. Further experiments revealed platelets acted as a bridge to mediate leukocyte adhesion, and platelet-mediated adhesion was the predominant pattern in the presence of platelets. However, platelet pre-incubation significantly suppressed leukocyte transendothelial migration after hypoxia-reoxygenation (over 31\%), which could be aggravated by blockade of endothelial-cell-expressed adhesion molecules, but alleviated by blockade of platelet-expressed adhesion molecules. This would indicate that platelet-mediated adhesion disrupted leukocyte transendothelial migration. An in vivo mesenteric ischemia-reperfusion model demonstrated leukocyte transfusion alone caused mild leukocyte adhesion to reperfused vessels and subsequent leukocyte infiltration, while simultaneous leukocyte and platelet transfusion led to massive leukocyte adhesion and sequestration within reperfused microvessels. Our studies revealed platelets enhanced leukocyte adhesion to endothelial cells, but suppressed leukocyte transendothelial migration. Overall, this leads to leukocyte sequestration in hypoxia-reoxygenated microvessels.
\end{abstract}

adhesion, endothelial cells, hypoxia-reoxygenation, leukocytes, platelets, transendothelial migration

Citation: Zheng, S., Cao, Y., Zhang, W., Liu, H., You, J., Yin, Y., Liu, J., and Li, C. (2016). Platelet-mediated adhesion facilitates leukocyte sequestration in hypoxia-reoxygenated microvessels . Sci China Life Sci 59, 299-311. doi: 10.1007/s11427-015-4986-1

\section{INTRODUCTION}

During an inflammatory response, leukocytes are first recruited to the site of injury and then adhere to the endothelium (Muller, 2011). However, subsequent consequences may vary. In some conditions or diseases, for example, multiple sclerosis, most leukocytes transmigrate through the endothelial barrier and infiltrate into the adjacent tissues, causing lesions in the affected area (Compston and Coles,

*Corresponding author (email: chenghui_li@sina.com)
2008). On the contrary, as in cerebral malaria, a few leukocytes transmigrate through the endothelium, but most are sequestrated within the microvessels, thus inducing local ischemia (Coltel et al., 2004; Pai et al., 2014). Yet, the reasons leading to leukocyte transmigration or sequestration in different conditions are poorly understood.

Ischemia-reperfusion (I-R) injury is a common complication during ischemic disease therapy and organ transplantation, in which leukocytes infiltrate into reperfused tissues and cause tissue lesions (Francischetti et al., 2010). Both leukocyte transendothelial migration (TEM) and sequestra- 
tion can be observed in I-R injury. After reperfusion, a variety of bioactive substances, especially chemokines, are produced. This leads to the recruitment of leukocytes to the site of ischemia (Muller, 2013). Leukocytes roll over and adhere to the vascular endothelium, then transmigrate through the endothelial barrier and infiltrate into the ischemic tissues, thus releasing reactive oxygen species and cytokines, which can cause cell death and tissue damage (Francischetti et al., 2010; Maroszynska and Fiedor, 2000). Alternatively, in some circumstances, leukocytes are aggregated within the reperfused microvasculature, impairing microcirculation. This happening is referred to as no-reflow phenomenon (Kaul, 2014). The underlying forces leading to such different outcomes still remain unclear. However, the treatment of the monoclonal antibody against the leukocyte adhesion molecule alleviates I-R injury and reduces the no-reflow incidence (Duilio et al., 2001). The interventions against adhesion molecules on endothelial cells (ECs) also exhibit beneficial effects (Dulkanchainun et al., 1998; Garcia-Criado et al., 1995; Tsuchihashi et al., 2006; Wong et al., 1997). These results suggest that leukocyte adhesion to the endothelium is the initial power behind leukocyte TEM or sequestration.

Besides leukocytes, platelets also actively participate in I-R injury. Significantly increased amounts of platelets adhere to endothelium during I-R and the depletion of platelets diminishes the extent of I-R injury (Pak et al., 2010). Interestingly, there is a synergistic relationship between leukocytes and platelets. Simultaneous reperfusion of platelets and leukocytes aggravates I-R injury more than does reperfusion of platelets or leukocytes alone (Sindram et al., 2001). It is noteworthy that platelets express various kinds of adhesion molecules which mediate the adhesion of platelets to numerous cell types. Such cell types include leukocytes and ECs (Ruggeri and Mendolicchio, 2007). Upon stimulation, these adhesion molecules are transported to the platelet surface and become activated (Ruggeri et al., 2006). Since both TEM and sequestration of leukocytes rely on its adhesion to the endothelium, platelets may have a great impact in determining leukocyte TEM or sequestration. In the present study, we examined the effects of platelets on leukocyte adhesion to ECs and TEM. We attempted to reveal the role of platelets in the determination of leukocyte TEM or sequestration in hypoxia-reoxygenation (H-R) condition.

\section{RESULTS}

\section{The effects of platelets on leukocyte adhesion to ECs after H-R}

First, we examined the effects of platelets on leukocyte adhesion to ECs. In our experiments, we employed primary cultured human brain endothelial cells (HBECs) and human lung endothelial cells (HLECs), immortalized liver sinusoidal endothelial cell (LSEC) line, leukocytes and platelets freshly isolated from peripheral blood. In a resting state without an H-R treatment, the leukocytes rarely adhered to ECs either with or without platelets. However, after the H-R treatment, the leukocyte adhesion was enormously increased compared with that of the resting groups. In the H-R-treated groups with platelet pre-incubation, leukocyte adhesion was further enhanced compared with that in the $\mathrm{H}-\mathrm{R}$ groups without platelet pre-incubation (Figure $1 \mathrm{~A}$ and B). This phenomenon was observed in all three kinds of ECs, indicating it was common for platelets to enhance leukocyte adhesion to ECs after an H-R treatment.

We further examined the adhesion molecules responsible for leukocyte adhesion under the H-R condition with or without platelets. We selected typical adhesion molecules expressed on ECs or platelets as blocking targets, namely CD36, intercellular adhesion molecule-1 (ICAM-1), vascular cell adhesion molecule-1 (VCAM-1), endothelial leukocyte adhesion molecule-1 (ELAM-1), glycoprotein Ib (gpIb), glycoprotein IIb (gpIIb), glycoprotein IIIa (gpIIIa), $\mathrm{P}$-selectin, and CD31. In the H-R condition without platelets, the antibody against ICAM-1, VCAM-1, ELAM-1, P-selectin, or CD31 significantly inhibited the adhesion of leukocytes to ECs, while CD36, gpIb, gpIIb, or gpIIIa blockade showed no significant effects (Figure 1C). In the H-R condition with platelets, the antibody against gpIb, gpIIb, gpIIIa, P-selectin, or CD31 significantly inhibited the adhesion of leukocytes to ECs, while CD36, ICAM-1, VCAM-1, or ELAM-1 blockade showed no significant effects (Figure 1D).

\section{The platelet adhesion to LSECs or leukocytes after H-R}

Platelets altered the relevant adhesion molecules mediating leukocyte adhesion to ECs. To reveal the underlying mechanism, we further examined platelet adhesion to ECs or leukocytes respectively. After the H-R treatment, the platelet adhesion to LSECs was significantly increased compared with that of the resting group (fluorescence intensity: $(142.1 \pm 7.5)$ of the resting versus $(289.2 \pm 20.0)$ of the H-R group; Figure $2 \mathrm{~A}$ and $\mathrm{B}$ ). The platelet adhesion to leukocytes was also enhanced after the H-R treatment (mean fluorescence intensity: $(5,550 \pm 1,570)$ of the resting versus $(14,028 \pm 2,019)$ of the H-R group; Figure $2 \mathrm{C}$ and D). The adhesion molecule blockade of gpIb, gpIlb, gpIIIa, P-selectin, or CD31 significantly inhibited platelet adhesion to LSECs, but the antibody against CD36, ICAM-1, VCAM-1, or ELAM-1 exhibited no significant effects (Figure 2E). Moreover, the relevant adhesion molecules responsible for plateletleukocyte adhesion were gpIb, gpIIb, gpIIIa, and P-selectin (Figure 2F). These results indicated that the positive effects of platelets on leukocyte adhesion to ECs might be due to the enhancement of platelet adhesion to ECs and leukocytes, with platelets acting as an intermediary bridge.

\section{The morphological evidences for leukocyte adhesion to ECs}

To confirm the intermediary role of platelets, we intensively 

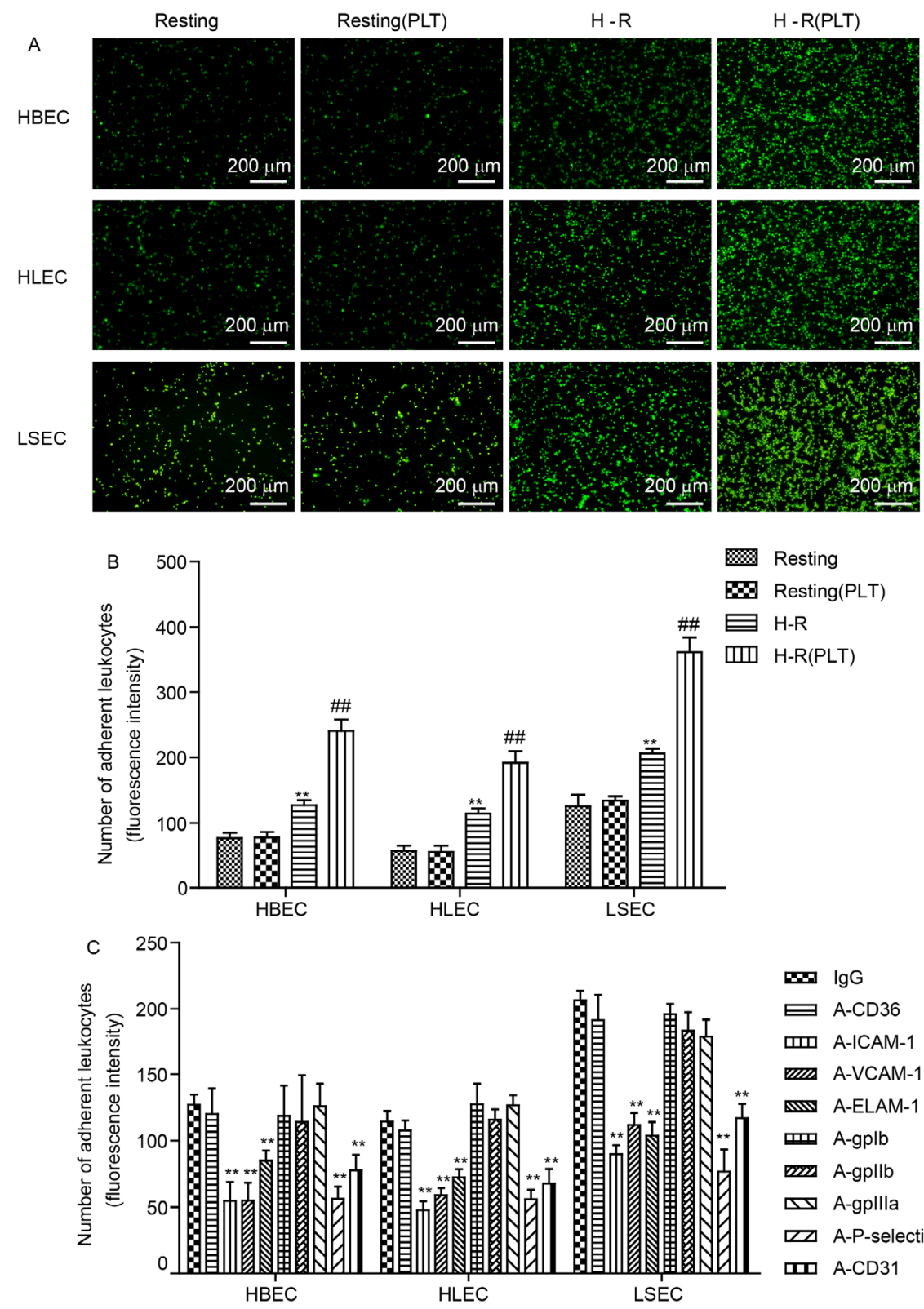

IgG

Е A-CD36

III A-ICAM-1

III A-VAM-1

A-ELAM-1

A-gplb

Z7z A-gpllb

A-gpllla

a-P-selectin

m A-CD31

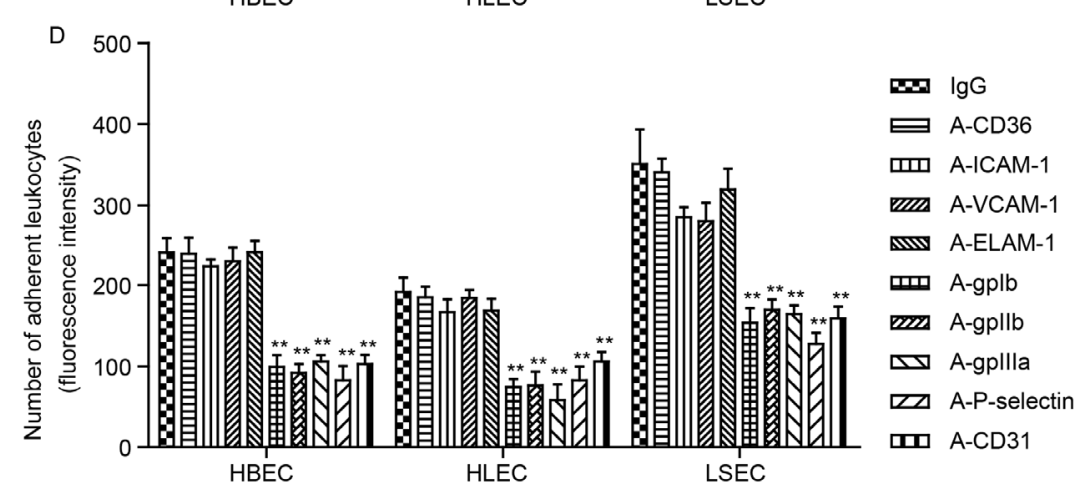

Figure 1 The effects of platelets on leukocyte adhesion to ECs after hypoxia-reoxygenation (H-R). A, Observation of the adhesion of BCECF-labeledleukocytes to human brain endothelial cells (HBECs), human lung endothelial cells (HLECs) and liver sinusoidal endothelial cells (LSECs) under a fluorescence microscope $(\times 100$; green fluorescence denoted leukocytes and PLT indicated platelet). B, The fluorescence intensity of the adherent leukocytes $(* *$, $P<0.01$, the H-R group versus the resting group; \#\#, $P<0.01$, the H-R (PLT) group versus the H-R group; $n=3$ ). C, The effects of the adhesion molecule blockade on the adhesion of leukocytes to ECs without platelets after $\mathrm{H}-\mathrm{R}$ (**, $P<0.01$, the antibody group versus the IgG group; $n=3$ ). $\mathrm{D}$, The effects of the adhesion molecule blockade on the adhesion of leukocytes to ECs with platelet pre-incubation after $\mathrm{H}-\mathrm{R}(* *, P<0.01$, the antibody group versus the IgG group; $n=3)$. 

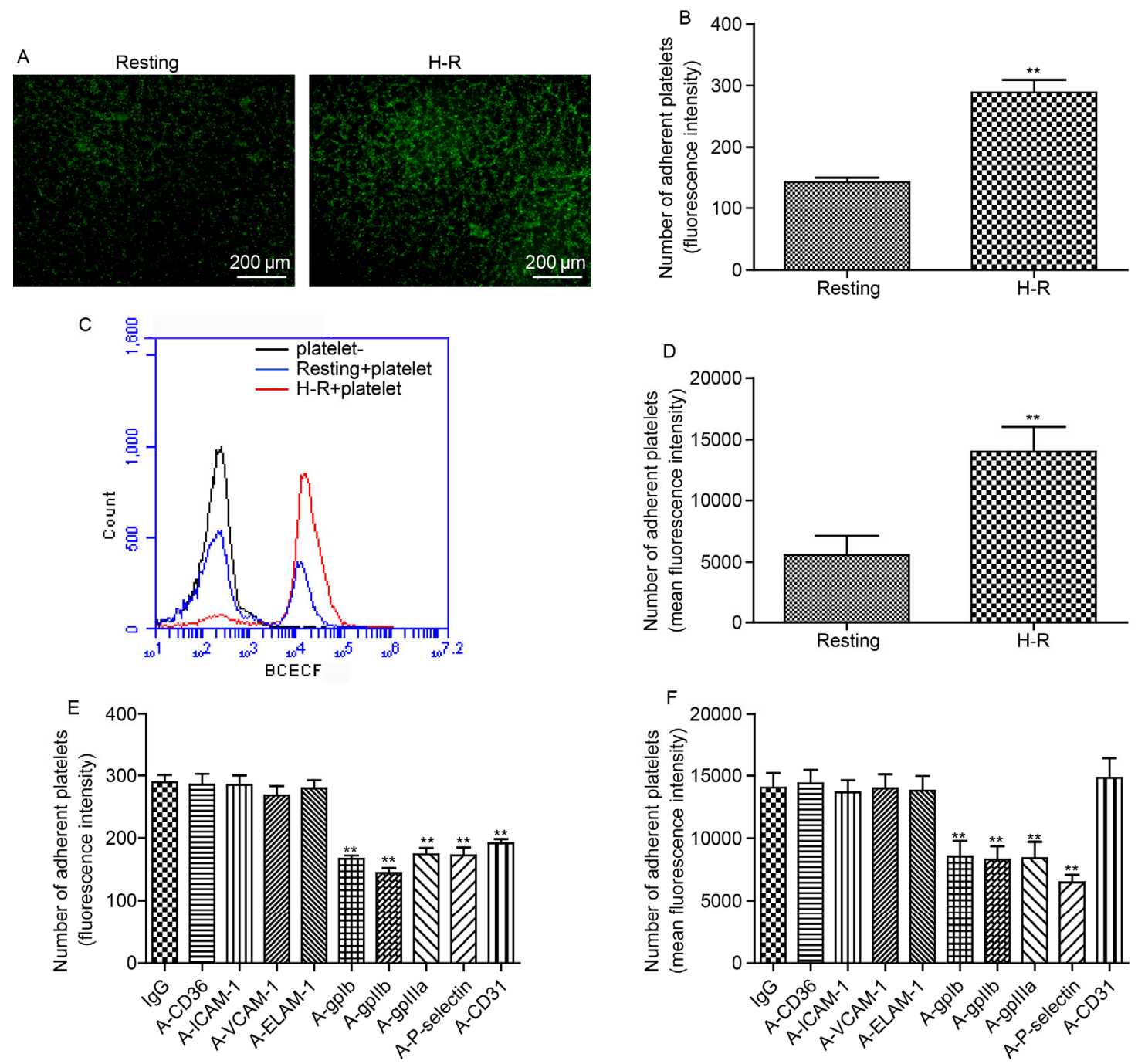

Figure 2 The platelet adhesion to LSECs or leukocytes after H-R. A, The adhesion of BCECF-labeled-platelets to a confluent LSEC monolayer observed by a fluorescence microscope $(\times 100$; green fluorescence denoted platelets). B, The fluorescence intensity of the adherent platelets $(* *, P<0.01$ compared with the resting group; $n=3$ ). C, The flow cytometry analysis of the adherent BCECF-labeled platelets to leukocytes after H-R. The leukocytes without platelet incubation were employed as a negative control. D, The mean fluorescence intensity of the adherent BCECF- labeled platelets to leukocytes after H-R (**, $P<0.01$ compared with the resting group; $n=3$ ). E, The effects of the adhesion molecule blockade on the adhesion of platelets to LSECs after H-R (**, $P<0.01$, the antibody group versus the IgG group; $n=3)$. F, The effects of the adhesion molecule blockade on the adhesion of platelets to leukocytes after H-R (**, $P<0.01$, the antibody group versus the $\mathrm{IgG}$ group; $n=3$ ).

observed leukocyte adhesion to ECs under a confocal or a transmission electron microscope. Under the H-R condition without platelets, the leukocytes directly adhered to the LSEC, as the confocal microscope observation evidenced (Figure 3A). However, under the H-R condition with platelets, considerably more leukocytes adhered to the LSEC (adherent leukocyte number: $(2.4 \pm 1.1)$ of the H-R versus $(6.8 \pm 0.8)$ of the H-R (PLT) group; Figure $3 \mathrm{~A}$ and B), and the majority of leukocyte adhesion was mediated by platelets (percentage of direct adhesion in the H-R (PLT) group: $(25.6 \% \pm 8.9 \%)$; Figure $3 \mathrm{~A}$ and $\mathrm{C})$. Further observation by an electron microscope also revealed that the leukocyte adhered to a LSEC by direct contact in the absence of platelets (Figure 3D). Conversely, under the H-R condition with platelets, the platelets protruded pseudopodia connecting the leukocyte and LSEC to mediate their adhesion. This revealed the intermediary role of platelets in mediating leukocyte adhesion to ECs (Figure 3D).

\section{The effects of platelets on leukocyte TEM after H-R}

Leukocyte adhesion to ECs is a preliminary step before leukocyte TEM. We next tried to reveal the effects of the platelet-enhanced leukocyte adhesion on leukocyte TEM after H-R. In a resting state, few leukocytes transmigrated through the EC layer no matter whether platelets were present or not. After the H-R treatment, significantly increased amounts of transmigrated leukocytes were observed in the groups without platelets compared to those in the groups of the resting state. However, in the H-R groups with platelet pre-incubation, the transmigrated leukocytes were signifi- 

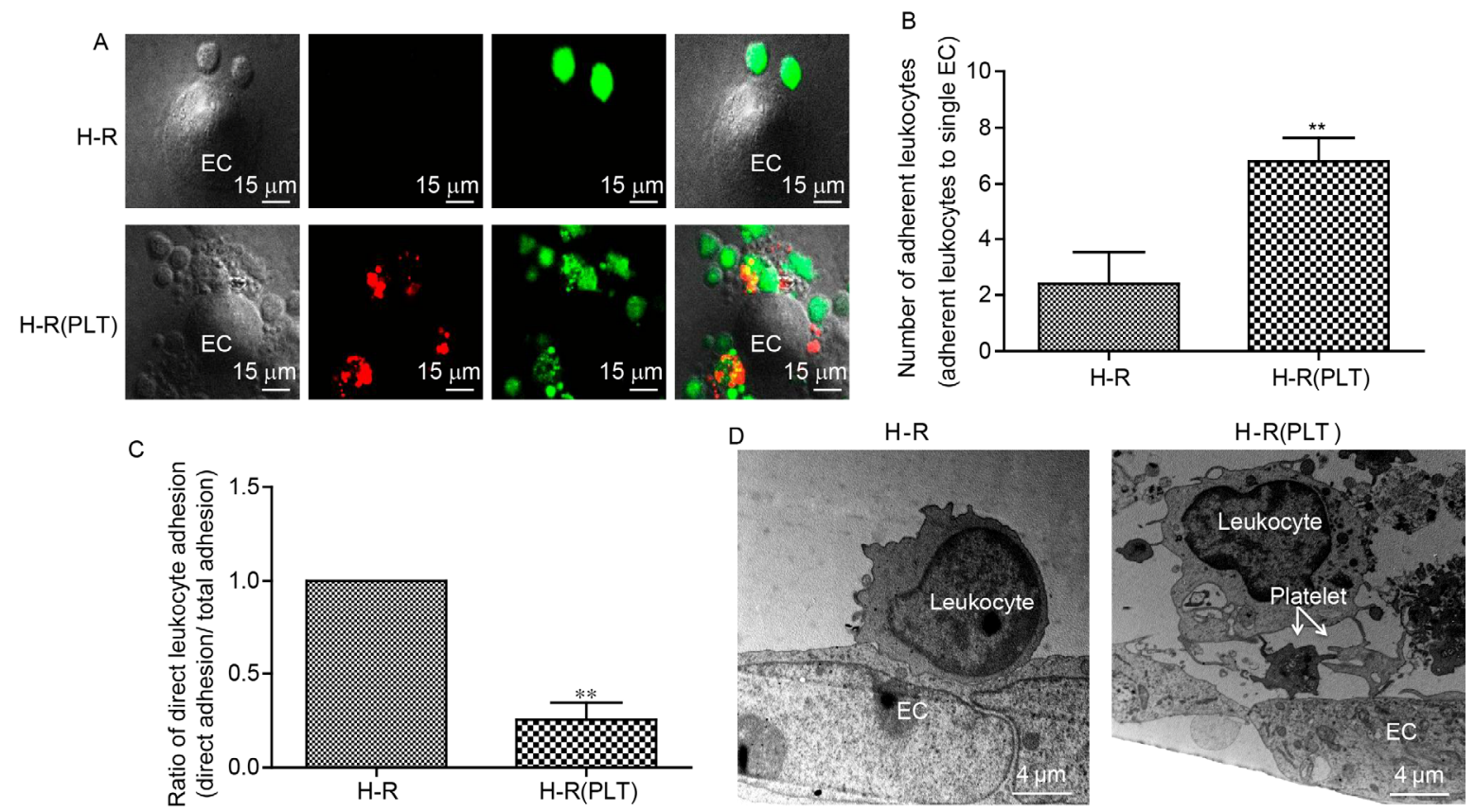

Figure 3 The morphological evidences for leukocyte adhesion to ECs. A, Observation of the leukocyte adhesion to LSEC under a laser scanning confocal microscope after H-R $(\times 1,800)$. The platelets were labeled with CM-DiI (red) and the leukocytes were labeled with BCECF (green). B, Quantification of the adherent leukocytes to a single LSEC ( $* *, P<0.01$ compared with the H-R group; $n=5$ ). C, Ratio of the direct adherent leukocytes to the total adherent leukocytes (**, $P<0.01$ compared with the H-R group; $n=5$ ). D, The transmission electron microscope images of the leukocyte adhesion to LSEC after H-R $(\times 8,000)$.

cantly fewer than those in the H-R groups without platelets (Figure 4A and B). Moreover, we examined the effects of adhesion molecule blockade on leukocyte TEM after the exposure to H-R. In the H-R groups without platelets, the antibody against ICAM-1, VCAM-1, ELAM-1, P-selectin, or CD31 significantly inhibited leukocyte TEM, while the antibody against CD36, gpIb, gpIIb, or gpIIIa showed no significant effects (Figure 4C). In the H-R groups with platelets, the antibody against ICAM-1, VCAM-1, or ELAM-1 further inhibited leukocyte TEM. On the other hand, the antibody against gpIb, gpIIb, or gpIIIa promoted leukocyte TEM, whereas the antibody against CD36, P-selectin, or CD31 exhibited no significant effects (Figure 4D).

\section{The effects of platelets on leukocyte adhesion and TEM in ischemic-reperfused vessels in vivo}

In vitro experiments demonstrated that platelets enhanced leukocyte adhesion to ECs but suppressed leukocyte TEM. To confirm the in vitro results, we conducted a series of in vivo experiments assessing the effects of platelets on adhesion and TEM of leukocytes in the I-R injury model. Healthy SD rats were employed to establish I-R in jejunum. After injecting the BCECF-labeled leukocytes with or without CM-DiI-labeled platelet pre-incubation, $10 \mathrm{~min}$ (acute) or $12 \mathrm{~h}$ (subacute) reperfusion was allowed. In the acute model, the injected leukocytes seldom adhered to mesenteric vessels regardless of whether platelets were injected or not in the non-ischemic area (Figure 5A). Con- versely, in the I-R area, leukocyte transfusion alone caused sporadic leukocyte adhesion, but the simultaneous transfusion of leukocytes and platelets induced massive leukocyte adhesion to the reperfused vessels (Figure 5A). Furthermore, in the subacute model, nearly no labeled leukocytes were found in the normoxia area (Figure 5B). In the I-R area, sporadic labeled leukocytes were distributed in the adjacent tissues rather than within the vessels after leukocyte transfusion alone, indicating that leukocyte TEM was the main outcome. Conversely, large amounts of labeled leukocytes were located within the reperfused vessels after the simultaneous transfusion of leukocytes and platelets. This revealed that platelets inhibited leukocyte TEM and induced leukocyte sequestration within the microvessels (Figure 5B). It was worthy of attention that the labeled platelets were co-localized with the labeled leukocytes after the simultaneous transfusion, thus confirming the intermediary role of platelets (Figure 5A and B).

\section{DISCUSSION}

In the present study, we provided three concepts confirmed by our experiments: (i) Platelets promoted leukocyte adhesion to ECs under H-R condition; (ii) platelets suppressed leukocyte TEM under H-R condition; (iii) platelets exerted a critical action in determining leukocyte TEM or sequestration in I-R injury.

In our experiments, the influence of platelets on leukocyte adhesion to ECs was demonstrated in three types of 

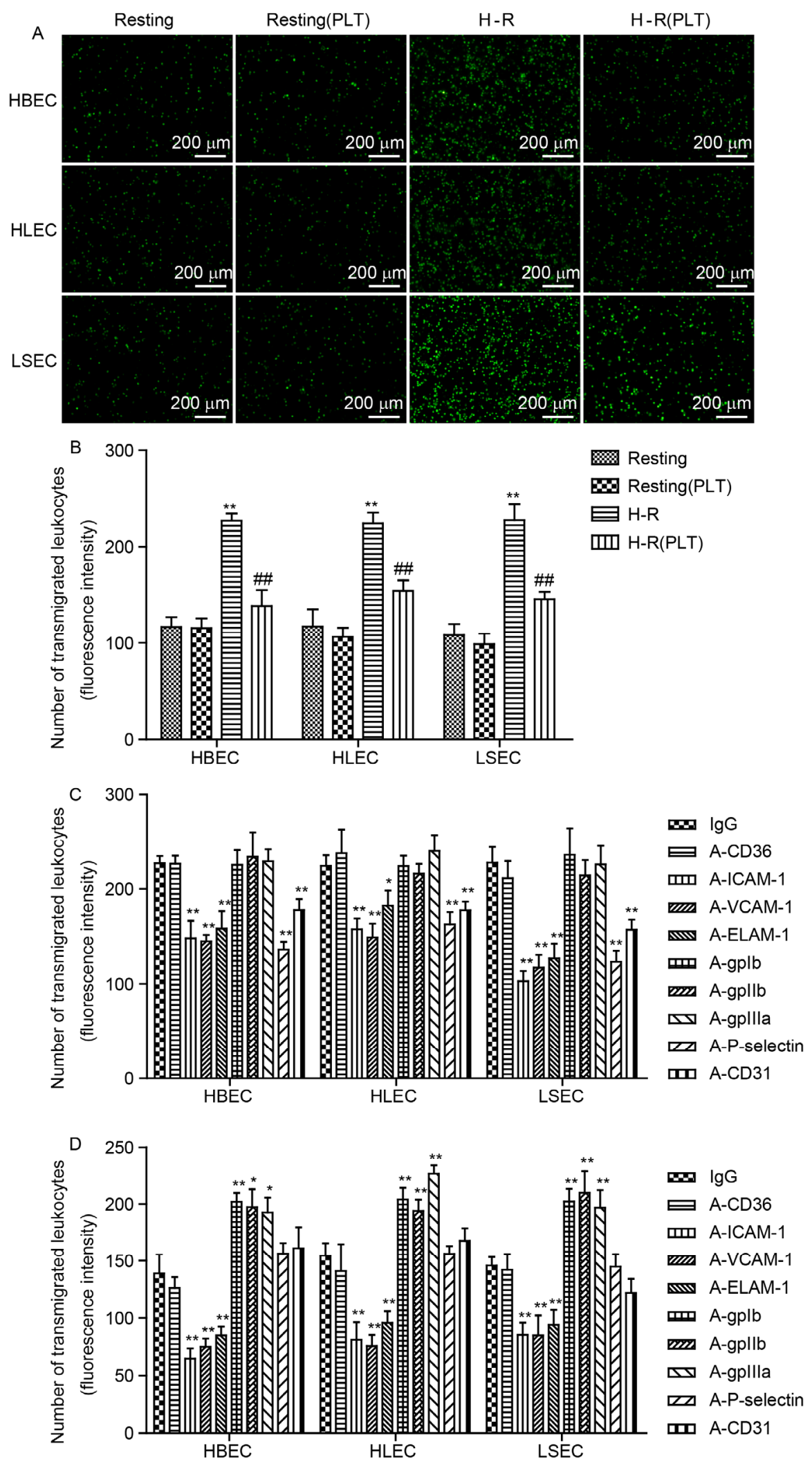

Figure 4 The effects of platelets on leukocyte TEM after H-R. A, Observation of the transmigrated leukocytes through the endothelial cell layer under a fluorescence microscope $(\times 100$; green fluorescence denoted leukocytes $)$. B, Quantification of the fluorescence intensity of the transmigrated leukocytes $(* *$, $P<0.01$, the H-R group versus the resting group; \#\#, $P<0.01$, the H-R (PLT) group versus the H-R group; $n=3$ ). C, The effects of the adhesion molecule blockade on leukocyte TEM without platelets after H-R $(*, P<0.05 ; * *, P<0.01$, the antibody group versus the IgG group; $n=3)$. D, The effects of the adhesion molecule blockade on leukocyte TEM with platelet pre-incubation after $\mathrm{H}-\mathrm{R}(*, P<0.05 ; * *, P<0.01$, the antibody group versus the IgG group; $n=3)$. 

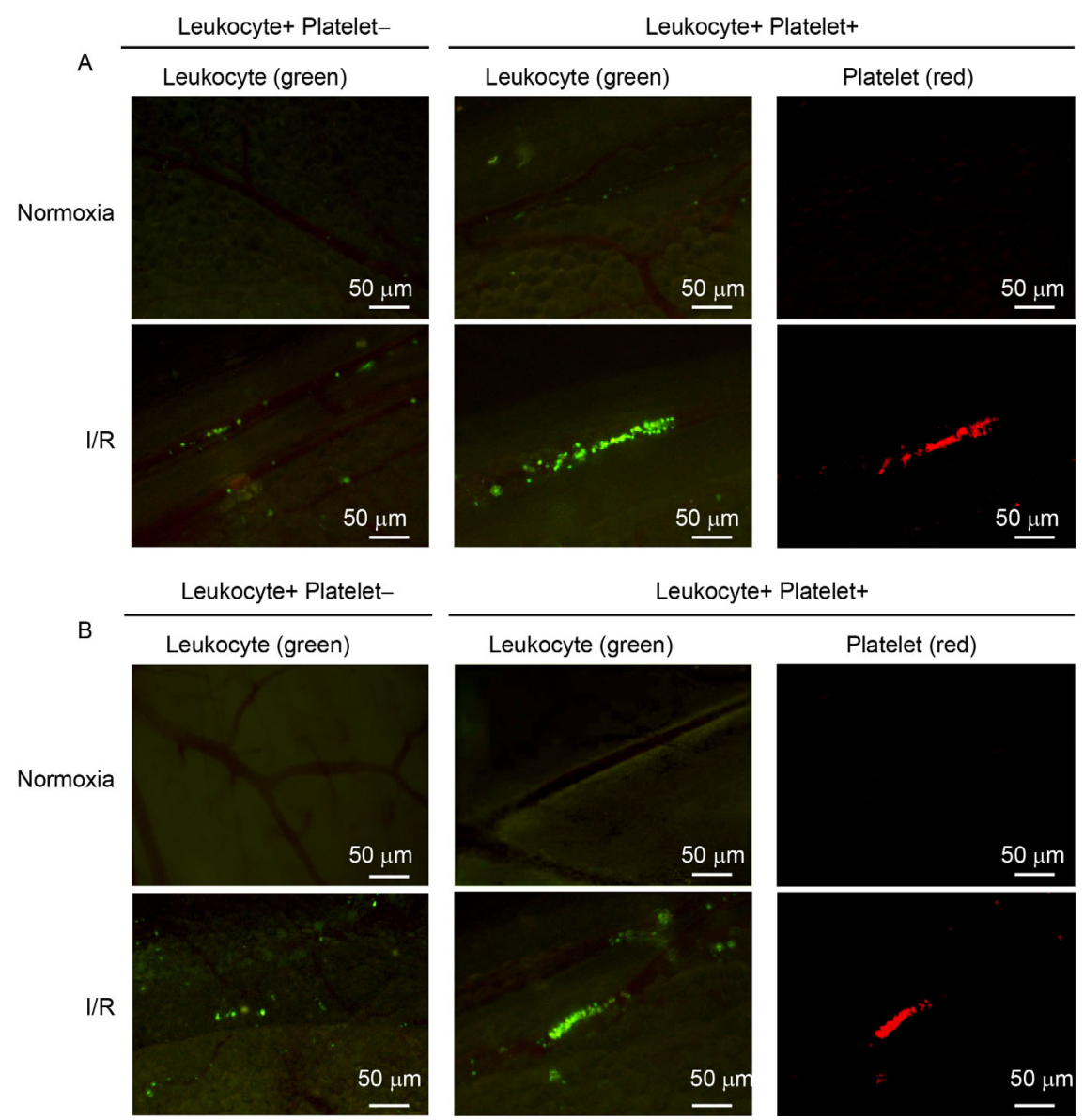

Figure 5 The effects of platelets on leukocyte adhesion and TEM in ischemic-reperfused vessels in vivo. The leukocytes were labeled with green fluorescent dye BCECF, and the platelets were labeled with red fluorescent dye CM-DiI. The H-R-treated leukocytes incubated with or without platelets were injected into the jejunal artery after one of its branches suffered ischemia for $30 \mathrm{~min}$. A, In the acute model (10 min after the transfusion), the leukocyte or platelet distribution was demonstrated by green or red fluorescence in the normoxia or ischemia-reperfused area $(n=10)$. B, In the subacute model $(12 \mathrm{~h}$ after the transfusion), the leukocyte or platelet distribution was demonstrated by green or red fluorescence in the normoxia or ischemia-reperfused area $(n=10)$.

ECs, two of primary cultured ECs and one immortalized endothelial cell line. In all three kinds of ECs, platelets further enhanced the H-R-induced leukocyte adhesion to ECs. In some cases other than I-R injury, there are also reports concerning platelet-dependent leukocyte adhesion. During the development of atherosclerosis, platelets adhere to the endothelium prone to injury and enhance the capability of endothelium to recruit leukocytes, thus contributing to atherosclerosis formation (Huo et al., 2003; May et al., 2007). The lymphocyte delivery to high endothelial venules also depends on platelet-induced adhesion (Diacovo et al., 1996a). A similar phenomenon is observed in inflamed brain microvessels, where leukocyte adhesion is mediated by platelets (Carvalho-Tavares et al., 2000). However, previous reports favor the view that platelets preferentially adhere to the subendothelial matrix, mediating the adhesion of leukocytes to the exposed subendothelial matrix rather than to ECs (Diacovo et al., 1996b; Kuijper et al., 1996). When our studies revealed that platelets enhanced leukocyte adhesion to ECs after the H-R treatment, we then focused on the differences of direct leukocyte adhesion and platelet- mediated leukocyte adhesion.

The adhesion of leukocytes to ECs is mediated by adhesion molecules, and the typical adhesion molecules we selected were expressed on ECs, such as ICAM-1, VCAM-1, and ELAM-1, or expressed on platelets, including gpIb, gpIIb, and gpIIIa, or even expressed on both (CD36, P-selectin, and CD31) (Zabel et al., 2015). CD36 showed no significant effects in any process in our study, indicating it did not participate in leukocyte adhesion or TEM. Except for CD36, it was apparent that the adhesion molecules expressed on ECs took part in leukocyte direct adhesion to ECs, while the adhesion molecules expressed on platelets participated in the platelet-mediated leukocyte adhesion. Further analyses evidenced that platelets adhered to both ECs and leukocytes, acting as a bridge to mediate their adhesion.

It has been reported that the use of H-R can activate ECs, enhance adhesion molecules expression, change the activity of coagulation and fibrinolysis, and produce cytokines, and thus recruit platelets and induce platelet adhesion (Yang et al., 2002). The adherent platelets could further fuse into 
ECs and support endothelium function (Wojcik et al., 1969), and thus play an important role in tumor necrosis factor (TNF)-induced microvascular pathology (Grau et al., 1993; Lou et al., 1997). Under some pathological conditions such as stroke and inflammation induced by cigarette smoking, leukocytes are not found as single cells, but as aggregates linked by activated platelets (Lehr, 2000; Ritter et al., 2005). Other reports find that P-selectin is crucial for platelet-leukocyte adhesion. The initial contact between platelets and leukocytes is driven by the recognition of $\mathrm{P}$-selectin on the platelet surface and P-selectin glycoprotein ligand-1 (PSGL-1) on the leukocyte surface. Such circumstances can trigger the conformational changes of integrins and further augment the adhesive capacity of leukocytes (da Costa Martins et al., 2006; Piccardoni et al., 2001). The binding of P-selectin to PSGL- 1 could also induce a series of inflammatory responses, including nuclear translocation of NF- $\kappa \mathrm{B}$ and the gene expression of various inflammatory cytokines, which is essential for the inflammatory phenotype acquisition of leukocytes (Dixon et al., 2006; Weyrich et al., 1996). The processes of platelet interaction with ECs and leukocytes are complicated and involve a great deal of conformational and functional alterations, which may contribute to the augmented leukocyte adhesion to ECs.

According to some traditional viewpoints, the firm adhesion of leukocytes to ECs is necessary for leukocyte TEM (Coito, 2011; Muller, 2011). In our studies, the H-R treatment without platelets increased leukocyte adhesion and subsequently increased leukocyte TEM. However, the enhanced leukocyte adhesion to ECs mediated by platelets did not promote, but suppressed leukocyte TEM. As we outlined above, previous studies focused on the adhesion of platelets and extracellular matrix. It has been reported that leukocytes can transmigrate through the platelet layer adhered to coated fibronectin by crosstalk of the adhesion molecules. This implies that platelets can facilitate leukocyte transmigration (Diacovo et al., 1996b). Nonetheless, leukocyte TEM is a much more complicated process, including adhesion molecule activation, cytoskeleton alteration, intercellular junction dissociation, and extracellular matrix enzyme secretion (Alcaide et al., 2008; Shaw et al., 2004; Sternlicht and Werb, 2001; Yang et al., 2006). Platelets may participate in any process and interfere with leukocyte TEM. We found that ICAM-1, VCAM-1, ELAM-1, P-selectin, and CD31 were not only significant to leukocyte adhesion, but also to leukocyte TEM in the platelet-free condition, thus indicating that the direct adhesion of leukocytes to ECs was necessary for leukocyte TEM. We hypothesized that the platelet-mediated adhesion altered some functional properties acquired by leukocyte direct adhesion to ECs and disrupted the subsequent TEM. Based on this hypothesis, the phenomena observed in our studies could be explained. Under the $\mathrm{H}-\mathrm{R}$ condition with platelets, the blockade of gpIb, gpIIb, or gpIIIa inhibited the platelet-mediated adhesion, and thus competitively increased leukocyte direct adhesion to ECs and the subsequent TEM. On the contrary, the blockade of ICAM-1, VCAM-1, or ELAM-1 further inhibited the leukocyte direct adhesion to ECs and suppressed leukocyte TEM. P-selectin and CD31 participated in both leukocyte direct adhesion and the platelet-mediated adhesion, so that the blockade of P-selectin or CD31 showed no significant effects, for the counteraction of both processes. This hypothesis was consistent with our in vitro results, and then we intensively tested the hypothesis in vivo.

In vivo, a mesenteric I-R model was established, due to superficial location and transparency of the mesenteric vessels, which allowed the direct observation of leukocyte distribution by fluorescent labeling. Since leukocyte adhesion was achieved within $10 \mathrm{~min}$ and leukocyte TEM occurred within $12 \mathrm{~h}$ after the initial adhesion (Zabel et al., 2015), the two phases were distinguished by the two time points. Leukocyte transfusion alone caused mild leukocyte adhesion in the acute phase and leukocyte TEM in the subacute phase. However, the simultaneous transfusion of leukocytes and platelets induced significant leukocyte adhesion in the acute phase and remarkable leukocyte sequestration in the subacute phase. These results were in agreement with our hypothesis and indicated that, at least in our experimental conditions, platelets determined the distinct outcomes of leukocyte TEM or sequestration.

Both leukocyte TEM and sequestration are harmful in pathological conditions. Leukocyte TEM is the preliminary step for leukocyte infiltration, which is the marker of inflammatory severity and tissue damage. Leukocyte sequestration can obstruct vessel lumen and induce local ischemia. Anti-platelet intervention may alleviate leukocyte sequestration, but otherwise extend leukocyte infiltration, a condition which raises concerns for the application of the anti-platelet therapy. The current anti-platelet therapy focuses on acute coronary syndromes. In clinical trials, anti-platelet drugs (such as aspirin and thienopyridines) can reduce the no-reflow occurrence after coronary intervention. Such a condition is considered to be attributed to their anti-coagulation effects (Mehta et al., 2008; Niccoli et al., 2010). Here, we demonstrated that the beneficial effects of the anti-platelet treatment might also include its suppression of leukocyte adhesion and sequestration. In summary, our studies revealed that platelets promoted leukocyte adhesion to ECs, but suppressed leukocyte TEM under H-R condition. The platelet-mediated leukocyte adhesion contributed to leukocyte sequestration in the microvasculature during the I-R injury (as illustrated in Figure 6).

\section{MATERIALS AND METHODS}

\section{Main reagents and equipment}

The cells were cultured in Dulbecco's modified eagle's medium (DMEM; Gibco, USA), and stained with the fluores 


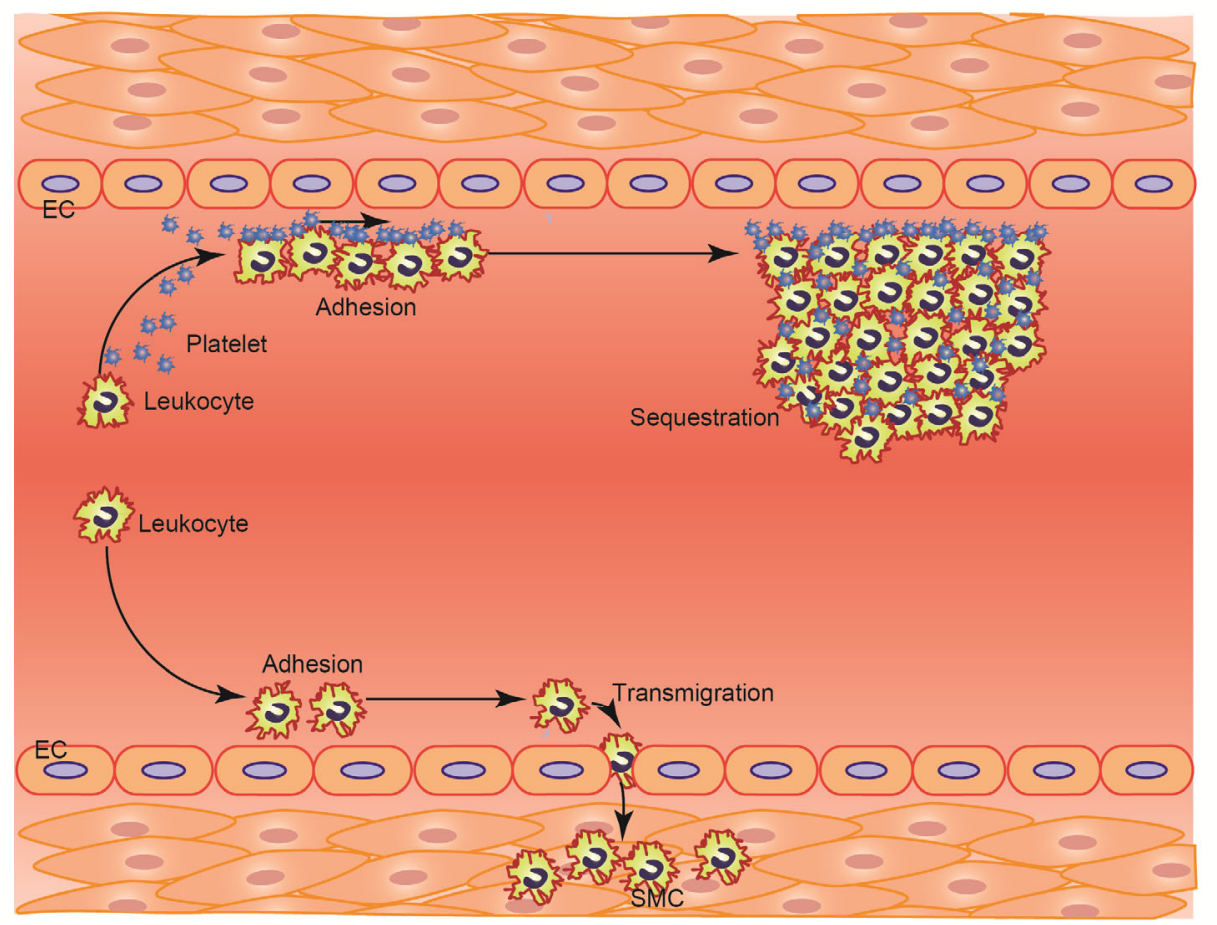

Figure 6 A schematic diagram of the influence of platelets on leukocyte TEM and sequestration. Under the H-R condition without platelets, the leukocytes adhered to ECs and subsequently transmigrated through the endothelium. Under the H-R condition with platelets, the leukocyte adhesion was enhanced, but the leukocyte TEM was suppressed, leading to leukocyte sequestration in hypoxia-reoxygenated microvessels.

cent dye CM-DiI (Invitrogen, USA) or 2',7'-bis-(2-carboxyethyl)-5-(and-6)-carboxyfluorescein (BCECF; Invitrogen, USA). Human or rat leukocytes were separated by human or rat leukocyte isolation kits (TBD Science, China). The murine monoclonal or polyclonal antibodies had specificity against human glycoprotein Ib (gpIb), glycoprotein IIb (gpIIb), glycoprotein IIIa (gpIIIa), P-selectin, CD31, CD36 (Chemicon, USA), intercellular adhesion molecule-1 (ICAM-1), vascular cell adhesion molecule-1 (VCAM-1), and endothelial leukocyte adhesion molecule-1 (ELAM-1) (Sigma, USA). The platelets were incubated with $10 \mathrm{nmol} \mathrm{L}^{-1}$ colloidal gold-labeled goat-anti-mouse antibody (Sigma, USA) for electron microscope observation. Images were acquired by using a fluorescence microscope (Olympus, Japan), a laser scanning confocal microscope (Leica, Germany) or a Type JEM 1010 transmission electron microscope (JEOL, Japan). The fluorescence intensity was quantified via a fluorescence plate reader (Molecular Devices, USA). The BCECF-labeled platelets adherence to leukocytes was detected by flow cytometry (Becton-Dickinson, USA).

\section{Isolation and purification of microvascular endothelial cells from human tissues}

Microvascular endothelial cells of human brains and lungs were isolated from autopsy donors without diseases of the central nervous system and lungs. The study protocol was examined and approved by the Ethical Committee for Re- search in Humans of China-Japan Friendship Hospital. The method for the isolation of microvascular endothelial cells was previously described (Wu et al., 2008). In brief, the tissues were cut into $2 \mathrm{~mm} \times 2 \mathrm{~mm}$ pieces and then digested in $10 \mathrm{~mL}$ of $0.2 \%$ collagenase type I (Sigma, USA) for 20 $\min$ at $37^{\circ} \mathrm{C}$. The samples were mixed with $5 \mathrm{~mL}$ of $0.1 \%$ trypsin plus $0.1 \%$ ethylene diamine tetraacetic acid (EDTA) and incubated for $5 \mathrm{~min}$ at $37^{\circ} \mathrm{C}$. Dulbecco's modified eagle's medium (DMED, $20 \mathrm{~mL}$ ) was then added to stop digestion. After passing through a $200-\mu \mathrm{m}$ filter, the cell suspension was collected and washed three times with DMEM. The pellet was suspended and incubated in DMEM containing 50\% fetal bovine serum (FBS) for 5 min to induce microvascular endothelial cell aggregation. The cell suspension was centrifuged at $500 \times \mathrm{g}$ for $5 \mathrm{~min}$, and the pellet was suspended in DMEM containing $2 \mathrm{mmol} \mathrm{L}^{-1} \mathrm{~L}$-glutamine, $100 \mathrm{U} \mathrm{mL}^{-1}$ penicillin, $100 \mu \mathrm{g} \mathrm{mL}^{-1}$ streptomycin, $20 \%$ FBS, $100 \mu \mathrm{g} \mathrm{mL}^{-1}$ endothelial cell growth supplement (ECGS), and $40 \mathrm{U} \mathrm{mL}^{-1}$ heparin. The cells were seeded in a 6-well plate coated with $2 \%$ gelatin type A (Sigma, USA). After 3-5 $d$ in culture, the cell colonies achieved the typical morphology of an endothelial cell colony. Under a phase contract microscope, the non-endothelial cells around the EC colonies were weeded out using a glass microtube. The remaining cells were digested and then seeded in a 6-well plate coated with $2 \%$ gelatin type A. The purified human endothelial cells at passages 5-8 were used in our study. The purity of microvascular endothelial cells was deter- 
mined and reported in our previous studies (Lou et al., 1998; Zhang et al., 2010b).

\section{Cell line}

A human liver sinusoidal endothelial cell (LSEC) line was established by transfecting primary cultures of human liver sinusoidal endothelial cells with simian vacuolating virus-40T and telomerase reverse transcriptase. The endothelial properties of LSEC were established in earlier investigations (Salmon et al., 2000; Zhang et al., 2010a).

\section{Isolation and fluorescent labeling of human platelets and leukocytes}

The human peripheral blood samples anti-coagulated with heparin were obtained from healthy volunteers. In the processes of platelet isolation, 1/10 volume of Acid Citrate Dextrose (ACD) solution (citric acid $0.038 \mathrm{~mol} \mathrm{~L}^{-1}$, citric acid trisodium $0.085 \mathrm{~mol} \mathrm{~L}^{-1}$, and glucose $0.136 \mathrm{~mol} \mathrm{~L}^{-1}$; $\mathrm{pH}=5.03$ ) was added to inhibit platelet activation. The samples were centrifuged at $270 \times g$ for $10 \mathrm{~min}$ at room temperature. The supernatant was collected and added to an equal volume of ACD solution. The samples were then centrifuged at $1,280 \times g$ for $10 \mathrm{~min}$ at room temperature, and the pellet was collected and washed three times with ACD solution to harvest the platelets. The separated platelets were used in the experiments immediately after isolation. Once the platelets were activated, a stage which could be distinguished by platelet aggregation, the platelets would be discarded to guarantee the platelets remained in a resting state before experiments.

In the processes of leukocyte isolation, leukocytes were separated by a human leukocyte isolation kit according to the manufacturer's instructions. In brief, blood samples were added above the separation medium layer, and then they were centrifuged at $400 \times g$ for $20 \mathrm{~min}$ at room temperature. Then, the leukocyte layers were collected and mixed with washing buffer. The samples were centrifuged at $250 \times g$ for $10 \mathrm{~min}$ at room temperature. The leukocyte pellet was collected and washed three times with washing buffer. The leukocyte samples were analyzed in the Department of Laboratory to examine the ratios of distinct leukocyte cell types. We chose samples with ratios in the physiologically normal range (neutrophils: 50\%-70\%; lymphocytes: $20 \%-$ 40\%; monocytes: $3 \%-10 \%$; eosinophils: $0.5 \%-5 \%$ ) so that the experiments could mimic the normal physiological condition. The freshly separated leukocytes were immediately utilized in the experiments to avoid leukocyte activation caused by long-time storage.

The isolated platelets and leukocytes were suspended in DMEM, and then incubated with the fluorescent dye CM-DiI or BCECF $\left(5 \mu \mathrm{g} \mathrm{mL}{ }^{-1}\right)$ at $37^{\circ} \mathrm{C}$ for $1 \mathrm{~h}$. After three washes, the labeled platelets and leukocytes were suspended in DMEM for further use.

\section{Hypoxia-reoxygenation (H-R) model establishment}

The cultured cells were incubated in an atmosphere of $95 \%$ $\mathrm{N}_{2}$ and $5 \% \mathrm{CO}_{2}$ at $37^{\circ} \mathrm{C}$ for $24 \mathrm{~h}$ in a closed container (hypoxia), and then they were transferred to an atmosphere of $95 \%$ air and $5 \% \mathrm{CO}_{2}$ at $37^{\circ} \mathrm{C}$ for $2 \mathrm{~h}$ (reoxygenation).

\section{Detection of leukocyte adhesion to endothelial cells}

The human brain endothelial cells (HBECs), human lung endothelial cells (HLECs) and liver sinusoidal endothelial cells (LSECs) were seeded on a 96-well plate and grew to confluence. In the experiments without platelets, the endothelial cells were treated with H-R, then BCECF-labeledleukocytes were added (leukocyte:endothelial cell=10:1), and the mixture was co-incubated for $30 \mathrm{~min}$. The nonadherent leukocytes were removed by three-fold washing. In the experiments with platelets, the endothelial cells were treated with $\mathrm{H}-\mathrm{R}$, then platelets were added, and the mixture was co-incubated for $4 \mathrm{~h}$ (platelet: endothelial cell=3,000:1). The non-adherent platelets were removed by washing three times. BCECF-labeled leukocytes were added (leukocyte: endothelial cell=10:1) and co-incubated for $30 \mathrm{~min}$, then the non-adherent leukocytes were removed by washing three times. Cell adhesion images were acquired by a fluorescence microscope and the fluorescence intensity was measured by a fluorescence plate reader. The groups without H-R treatment were set as controls.

In the antibody blocking experiments, endothelial cells were incubated with mouse anti-human gpIb, gpIIb, gpIIIa, P-selectin, CD31, CD36, ICAM-1, VCAM-1, or ELAM-1 antibody (final concentration $=5 \mu \mathrm{g} \mathrm{mL}{ }^{-1}$ ) before the coculture with platelets or leukocytes. The control groups were incubated with unrelated IgG.

\section{Detection of platelet adhesion to LSECs}

The LSECs were inoculated in a 96-well plate and grew to confluence. After the LSECs were subjected to an H-R treatment, BCECF-labeled platelets were added (platelet:LSEC=3,000:1) and co-incubated with H-R-treated LSECs for $4 \mathrm{~h}$. After three washes, the non-adherent platelets were removed. The images were recorded by a fluorescence microscope, and the fluorescence intensity was measured by a fluorescence plate reader. The LSECs grown in normoxia condition were set as a control.

In the antibody blocking experiments, mouse anti-human gpIb, gpIIb, gpIIIa, P-selectin, CD31, CD36, ICAM-1, VCAM-1, or ELAM-1 antibody was incubated with LSECs before the platelets were added (final concentration $=5$ $\mu \mathrm{g} \mathrm{mL}^{-1}$ ). The control groups were incubated with unrelated IgG.

\section{Detection of platelet adhesion to leukocytes}

The leukocytes were subjected to an H-R treatment and then were co-incubated with BCECF-labeled platelets (platelet: leukocyte $=3,000: 1)$ for $4 \mathrm{~h}$. The samples were centri- 
fuged at $270 \times g$ for $10 \mathrm{~min}$ at room temperature, and the non-adherent platelet supernatants were discarded. After three times of centrifugation and suspension, the pellet was suspended for a flow cytometry analysis. The leukocytes grown under normoxia condition were set as a normal control, and the leukocytes without platelet incubation were set as a negative control.

In the antibody blocking experiments, mouse anti-human gpIb, gpIIb, gpIIIa, P-selectin, CD31, CD36, ICAM-1, VCAM-1, or ELAM-1 antibody was incubated with leukocytes before the platelets were added (final concentration= $5 \mu \mathrm{g} \mathrm{mL}^{-1}$ ). The control groups were incubated with unrelated $\mathrm{IgG}$.

\section{Morphological observation of the leukocyte adhesion to LSECS}

In the laser scanning confocal microscope observation, the platelets were labeled with CM-DiI, and the leukocytes were labeled with BCECF. After LSECs were seeded into a Petri dish and grew to $50 \%$ confluence, similar processes were carried out as described in the subsection of "Detection of leukocyte adhesion to endothelial cells". The images were obtained by a laser scanning confocal microscope. In each group, five endothelial cells were randomly selected, and the leukocytes adhered to a single endothelial cell were counted. In the platelet-treated group, leukocyte direct adhesion or platelet-mediated adhesion was respectively counted, and the ratios of the direct adherent leukocytes to the total adherent leukocytes were calculated.

In the electron microscope observation, the LSECs were inoculated into a cell culture insert and grew to confluence. After the LSECs were subjected to an H-R treatment, the platelets were added to the top layer of the chamber and were co-incubated with LSECs for $4 \mathrm{~h}$ (platelet:LSEC= $3,000: 1)$. The non-adherent platelets were removed by three-fold washing. Then leukocytes were added (leukocyte:LSEC=10:1) and co-incubated for $30 \mathrm{~min}$. The non-adherent leukocytes were removed by washing three times. The samples were fixed in $1 \%$ paraformaldehyde and $0.125 \%$ glutaraldehyde for $30 \mathrm{~min}$. After three washes with phosphate buffered saline (PBS), the mouse anti-human gpIIb monoclonal antibody (final concentration of 5 $\mu \mathrm{g} \mathrm{mL}^{-1}$ ) was added and incubated with the samples overnight at $4^{\circ} \mathrm{C}$. After three washes with PBS, the colloidal gold-labeled goat-anti-mouse antibody (final concentration of $10 \mathrm{nmol} \mathrm{L}{ }^{-1}$ ) was added. After incubation at room temperature for $60 \mathrm{~min}$, the samples were washed three times with PBS and fixed with $2.5 \%$ glutaraldehyde for $30 \mathrm{~min}$. After three PBS washes, the samples were fixed with $1 \%$ osmic acid for $20 \mathrm{~min}$. The cell insert was removed after three washes with PBS, and the membrane was taken off along the bottom of the insert. After dehydration, the specimens were embedded in epoxy resin mixture and prepared for the transmission electron microscope observation. In the
H-R group without platelet pre-incubation, the same H-R and co-culture procedures as described in "Detection of leukocyte adhesion to endothelial cells" were conducted, and the samples were prepared for electron microscope observation as detailed above. The images were recorded by a transmission electron microscope.

\section{Detection of leukocyte transmigration through endothe- lial cell layer}

The HBECs, HLECs and LSECs were seeded on the inserts of migration chambers and grew to confluence. In the experiments with platelets, the endothelial cells were subjected to an H-R treatment, then the platelets were added to the upper layer of the migration chamber inserts, and the mixture were co-cultured for $30 \mathrm{~min}$ (platelet: endothelial cell= $3,000: 1)$. The non-adherent platelets were removed by washing three times. BCECF-labeled leukocytes were then added to the top layer (leukocyte: endothelial cell=10:1), while $100 \mathrm{U} \mathrm{mL}^{-1}$ interferon- $\gamma$ (Sigma, USA) and 1,000 $\mathrm{U} \mathrm{mL} \mathrm{L}^{-1}$ tumor necrosis factor- $\alpha$ (TNF- $\alpha$; Sigma, USA) were added to the medium in the lower chamber. The cells that had passed through the migration chamber insert were collected $12 \mathrm{~h}$ later, and were centrifuged and plated in a 96-well plate. The migrated cells were detected by a fluorescence microscope and measured by a fluorescence plate reader. In the experiments without platelets, similar procedures were conducted (as described above), except that no platelets were added before leukocyte co-incubation. Groups without H-R treatment were set as controls.

In the antibody blocking experiments, mouse anti-human gpIb, gpIIb, gpIIIa, P-selectin, CD31, CD36, ICAM-1, VCAM-1, or ELAM-1 antibody was incubated with endothelial cells before platelets or leukocytes were added (final concentration $=5 \mu \mathrm{g} \mathrm{mL}^{-1}$ ). The control groups were incubated with unrelated IgG.

\section{Establishment of the mesenteric ischemia-reperfusion model}

Ten-week-old male Sprague-Dawley rats were purchased from Vital River Laboratory Animal Inc. The research protocols were reviewed and approved by the Animal Care and Use Review Committee of China-Japan Friendship Hospital. The present study complied with the Guide for the Care and Use of Laboratory Animals published by the US National Institutes of Health (NIH publication no. 85-23, revised 1996). The rat platelets were separated according to the method mentioned for human platelet isolation with minor adjustments. The rat leukocytes were separated by a rat leukocyte isolation kit with procedures similar to those for human leukocyte isolation. The platelets were labeled with CM-DiI, and the leukocytes were labeled with BCECF. The leukocytes were subjected to an H-R treatment. Then the platelets and leukocytes were co-incubated for $30 \mathrm{~min}$ (platelet:leukocyte=3,000:1). The samples were centri- 
fuged at $270 \times g$ for $10 \mathrm{~min}$ at room temperature, and the non-adherent platelet supernatants were discarded.

The rats were anesthetized by a pentobarbital sodium intraperitoneal injection $\left(40 \mathrm{mg} \mathrm{kg}^{-1}\right)$. The abdominal wall was incised, and one branch of mesenteric artery was clamped for $30 \mathrm{~min}$. Then, the clamp was released, and $1 \times 10^{7}$ leukocytes with or without platelet incubation were injected into the jejunal artery, allowing them to flow through both the ischemic-reperfused and normal-perfused mesenteric branches. Ten rats were treated in the acute (10 min of reperfusion) model, and another ten were treated in the subacute ( $12 \mathrm{~h}$ of reperfusion) model. In the acute model, the affected vessels and their projected jejunum were observed under a fluorescence microscope $10 \mathrm{~min}$ after leukocyte injection. In the subacute model, the abdominal walls were closed after the leukocyte injection, and the rats were sedated by a low-dose intraperitoneal injection of pentobarbital sodium $\left(15 \mathrm{mg} \mathrm{kg}^{-1}\right)$, which was administered every $4 \mathrm{~h}$. Twelve hours after the leukocyte injection, the affected vessels and their projected jejunum were exposed and observed under a fluorescence microscope.

\section{Statistical analysis}

The data were analyzed with GraphPad Prism 5 software and expressed as mean \pm standard deviation. The differences between groups were compared by $t$-test (only two groups) or One-way ANOVA (more than two groups). $P<0.05$ was considered statistically significant.

Compliance and ethics The author(s) declare that they have no conflict of interest.

For studies involving human subjects and animals, the authors conformed with the Helsinki Declaration of 1975 (as revised in 2008) concerning Human and Animal Rights, and followed out policy concerning Informed Consent as shown in Springer.com.

Acknowledgements This work was supported by the Natural Science Foundation of Beijing City (7092093).

Alcaide, P., Newton, G., Auerbach, S., Sehrawat, S., Mayadas, T.N., Golan, D.E., Yacono, P., Vincent, P., Kowalczyk, A., and Luscinskas, F.W. (2008). p120-Catenin regulates leukocyte transmigration through an effect on VE-cadherin phosphorylation. Blood 112, 2770-2779.

Carvalho-Tavares, J., Hickey, M.J., Hutchison, J., Michaud, J., Sutcliffe, I.T., and Kubes, P. (2000). A role for platelets and endothelial selectins in tumor necrosis factor-alpha-induced leukocyte recruitment in the brain microvasculature. Circ Res 87, 1141-1148.

Coito, A.J. (2011). Leukocyte transmigration across endothelial and extracellular matrix protein barriers in liver ischemia/reperfusion injury. Curr Opin Organ Transplant 16, 34-40.

Coltel, N., Combes, V., Hunt, N.H., and Grau, G.E. (2004). Cerebral malaria-a neurovascular pathology with many riddles still to be solved. Curr Neurovasc Res 1, 91-110.

Compston, A., and Coles, A. (2008). Multiple sclerosis. Lancet 372, 1502-1517.

da Costa Martins, P.A., van Gils, J.M., Mol, A., Hordijk, P.L., and Zwaginga, J.J. (2006). Platelet binding to monocytes increases the adhesive properties of monocytes by up-regulating the expression and functionality of beta1 and beta2 integrins. J Leukoc Biol 79, 499-507.

Diacovo, T.G., Puri, K.D., Warnock, R.A., Springer, T.A., and von Andrian, U.H. (1996a). Platelet-mediated lymphocyte delivery to high endothelial venules. Science 273, 252-255.

Diacovo, T.G., Roth, S.J., Buccola, J.M., Bainton, D.F., and Springer, T.A. (1996b). Neutrophil rolling, arrest, and transmigration across activated, surface-adherent platelets via sequential action of P-selectin and the beta 2-integrin CD11b/CD18. Blood 88, 146-157.

Dixon, D.A., Tolley, N.D., Bemis-Standoli, K., Martinez, M.L., Weyrich, A.S., Morrow, J.D., Prescott, S.M., and Zimmerman, G.A. (2006). Expression of COX-2 in platelet-monocyte interactions occurs via combinatorial regulation involving adhesion and cytokine signaling. $\mathrm{J}$ Clin Invest 116, 2727-2738.

Duilio, C., Ambrosio, G., Kuppusamy, P., DiPaula, A., Becker, L.C., and Zweier, J.L. (2001). Neutrophils are primary source of $\mathrm{O}_{2}$ radicals during reperfusion after prolonged myocardial ischemia. Am J Physiol Heart Circ Physiol 280, H2649-H2657.

Dulkanchainun, T.S., Goss, J.A., Imagawa, D.K., Shaw, G.D., Anselmo, D.M., Kaldas, F., Wang, T., Zhao, D., Busuttil, A.A., Kato, H., Murray, N.G., Kupiec-Weglinski, J.W., and Busuttil, R.W. (1998). Reduction of hepatic ischemia/reperfusion injury by a soluble P-selectin glycoprotein ligand-1. Ann Surg 227, 832-840.

Francischetti, I., Moreno, J.B., Scholz, M., and Yoshida, W.B. (2010). Leukocytes and the inflammatory response in ischemia-reperfusion injury. Rev Bras Cir Cardiovasc 25, 575-584.

Garcia-Criado, F.J., Toledo-Pereyra, L.H., Lopez-Neblina, F., Phillips, M.L., Paez-Rollys, A., and Misawa, K. (1995). Role of P-selectin in total hepatic ischemia and reperfusion. J Am Coll Surg 181, 327-334.

Grau, G.E., Tacchini-Cottier, F., Vesin, C., Milon, G., Lou, J.N., Piguet, P.F., and Juillard, P. (1993). TNF-induced microvascular pathology: active role for platelets and importance of the LFA-1/ICAM-1 interaction. Eur Cytokine Netw 4, 415-419.

Huo, Y., Schober, A., Forlow, S.B., Smith, D.F., Hyman, M.C., Jung, S., Littman, D.R., Weber, C., and Ley, K. (2003). Circulating activated platelets exacerbate atherosclerosis in mice deficient in apolipoprotein E. Nat Med 9, 61-67.

Kaul, S. (2014). The "no reflow" phenomenon following acute myocardial infarction: Mechanisms and treatment options. J Cardiol 64, 77-85.

Kuijper, P.H., Gallardo Torres, H.I., van der Linden, J.A., Lammers, J.W., Sixma, J.J., Koenderman, L., and Zwaginga, J.J. (1996). Platelet-dependent primary hemostasis promotes selectin- and integrin-mediated neutrophil adhesion to damaged endothelium under flow conditions. Blood 87, 3271-3281.

Lehr, H.A. (2000). Microcirculatory dysfunction induced by cigarette smoking. Microcirculation 7, 367-384.

Lou, J., Donati, Y.R., Juillard, P., Giroud, C., Vesin, C., Mili, N., and Grau, G.E. (1997). Platelets play an important role in TNF-induced microvascular endothelial cell pathology. Am J Pathol 151, 1397-1405.

Lou, J.N., Mili, N., Decrind, C., Donati, Y., Kossodo, S., Spiliopoulos, A., Ricou, B., Suter, P.M., Morel, D.R., Morel, P., and Grau, G.E. (1998). An improved method for isolation of microvascular endothelial cells from normal and inflamed human lung. In Vitro Cell Dev Biol Anim 34, 529-536.

Maroszynska, I., and Fiedor, P. (2000). Leukocytes and endothelium interaction as rate limiting step in the inflammatory response and a key factor in the ischemia-reperfusion injury. Ann Transplant 5, 5-11.

May, A.E., Langer, H., Seizer, P., Bigalke, B., Lindemann, S., and Gawaz, M. (2007). Platelet-leukocyte interactions in inflammation and atherothrombosis. Semin Thromb Hemost 33, 123-127.

Mehta, S.R., Bassand, J.P., Chrolavicius, S., Diaz, R., Fox, K.A., Granger, C.B., Jolly, S., Rupprecht, H.J., Widimsky, P., Yusuf, S., and Committee, C.O.S. (2008). Design and rationale of CURRENT-OASIS 7: a randomized, $2 \times 2$ factorial trial evaluating optimal dosing strategies for clopidogrel and aspirin in patients with ST and non-ST-elevation acute coronary syndromes managed with an early invasive strategy. Am Heart J 156, 1080-1088 e1081.

Muller, W.A. (2011). Mechanisms of leukocyte transendothelial migration. Annu Rev Pathol 6, 323-344. 
Muller, W.A. (2013). Getting leukocytes to the site of inflammation. Vet Pathol 50, 7-22.

Niccoli, G., Spaziani, C., Marino, M., Pontecorvo, M.L., Cosentino, N., Baca, M., Porto, I., Leone, A.M., and Crea, F. (2010). Effect of chronic Aspirin therapy on angiographic thrombotic burden in patients admitted for a first ST-elevation myocardial infarction. Am J Cardiol 105, 587-591.

Pai, S., Qin, J., Cavanagh, L., Mitchell, A., El-Assaad, F., Jain, R., Combes, V., Hunt, N.H., Grau, G.E., and Weninger, W. (2014). Real-time imaging reveals the dynamics of leukocyte behaviour during experimental cerebral malaria pathogenesis. PLoS Pathog 10, e1004236.

Pak, S., Kondo, T., Nakano, Y., Murata, S., Fukunaga, K., Oda, T., Sasaki, R., and Ohkohchi, N. (2010). Platelet adhesion in the sinusoid caused hepatic injury by neutrophils after hepatic ischemia reperfusion. Platelets 21, 282-288.

Piccardoni, P., Sideri, R., Manarini, S., Piccoli, A., Martelli, N., de Gaetano, G., Cerletti, C., and Evangelista, V. (2001). Platelet/polymorphonuclear leukocyte adhesion: a new role for SRC kinases in Mac-1 adhesive function triggered by P-selectin. Blood 98, 108-116.

Ritter, L.S., Stempel, K.M., Coull, B.M., and McDonagh, P.F. (2005). Leukocyte-platelet aggregates in rat peripheral blood after ischemic stroke and reperfusion. Biol Res Nurs 6, 281-288.

Ruggeri, Z.M., and Mendolicchio, G.L. (2007). Adhesion mechanisms in platelet function. Circ Res 100, 1673-1685.

Ruggeri, Z.M., Orje, J.N., Habermann, R., Federici, A.B., and Reininger, A.J. (2006). Activation-independent platelet adhesion and aggregation under elevated shear stress. Blood 108, 1903-1910.

Salmon, P., Oberholzer, J., Occhiodoro, T., Morel, P., Lou, J., and Trono, D. (2000). Reversible immortalization of human primary cells by lentivector-mediated transfer of specific genes. Mol Ther 2, 404-414.

Shaw, S.K., Ma, S., Kim, M.B., Rao, R.M., Hartman, C.U., Froio, R.M., Yang, L., Jones, T., Liu, Y., Nusrat, A., Parkos, C.A., and Luscinskas, F.W. (2004). Coordinated redistribution of leukocyte LFA-1 and endothelial cell ICAM-1 accompany neutrophil transmigration. J Exp Med 200, 1571-1580.

Sindram, D., Porte, R.J., Hoffman, M.R., Bentley, R.C., and Clavien, P.A. (2001). Synergism between platelets and leukocytes in inducing endothelial cell apoptosis in the cold ischemic rat liver: a Kupffer cell-mediated injury. FASEB J 15, 1230-1232.
Sternlicht, M.D., and Werb, Z. (2001). How matrix metalloproteinases regulate cell behavior. Annu Rev Cell Dev Biol 17, 463-516.

Tsuchihashi, S., Fondevila, C., Shaw, G.D., Lorenz, M., Marquette, K., Benard, S., Shen, X.D., Ke, B., Busuttil, R.W., and Kupiec-Weglinski, J.W. (2006). Molecular characterization of rat leukocyte P-selectin glycoprotein ligand-1 and effect of its blockade: protection from ischemia-reperfusion injury in liver transplantation. J Immunol 176, 616-624.

Weyrich, A.S., Elstad, M.R., McEver, R.P., McIntyre, T.M., Moore, K.L., Morrissey, J.H., Prescott, S.M., and Zimmerman, G.A. (1996). Activated platelets signal chemokine synthesis by human monocytes. J Clin Invest 97, 1525-1534.

Wojcik, J.D., Van Horn, D.L., Webber, A.J., and Johnson, S.A. (1969). Mechanism whereby platelets support the endothelium. Transfusion 9 , 324-335.

Wong, J., Johnston, B., Lee, S.S., Bullard, D.C., Smith, C.W., Beaudet, A.L., and Kubes, P. (1997). A minimal role for selectins in the recruitment of leukocytes into the inflamed liver microvasculature. J Clin Invest 99, 2782-2790.

Wu, L.Q., Zhang, W.J., Niu, J.X., Ye, L.Y., Yang, Z.H., Grau, G.E., and Lou, J.N. (2008). Phenotypic and functional differences between human liver cancer endothelial cells and liver sinusoidal endothelial cells. J Vasc Res 45, 78-86.

Yang, H., Majno, P., Morel, P., Toso, C., Triponez, F., Oberholzer, J., Mentha, G., and Lou, J. (2002). Prostaglandin E(1) protects human liver sinusoidal endothelial cell from apoptosis induced by hypoxia reoxygenation. Microvasc Res 64, 94-103.

Yang, L., Kowalski, J.R., Zhan, X., Thomas, S.M., and Luscinskas, F.W. (2006). Endothelial cell cortactin phosphorylation by Src contributes to polymorphonuclear leukocyte transmigration in vitro. Circ Res 98, 394-402.

Zabel, B.A., Rott, A., and Butcher, E.C. (2015). Leukocyte chemoattractant receptors in human disease pathogenesis. Annu Rev Pathol 10, 51-81.

Zhang, B.H., Zhang, W.J., Lou, J.N., and Li, C.H. (2010a). Morphologic and functional characteristics of the immortalized human liver sinusoidal endothelial cell line. Zhonghua Wai Ke Za Zhi 48, 48-52.

Zhang, W.J., Feng, J., Zhou, R., Ye, L.Y., Liu, H.L., Peng, L., Lou, J.N., and Li, C.H. (2010b). Tanshinone IIA protects the human blood-brain barrier model from leukocyte-associated hypoxia-reoxygenation injury. Eur J Pharmacol 648, 146-152.

Open Access This article is distributed under the terms of the Creative Commons Attribution License which permits any use, distribution, and reproduction in any medium, provided the original author(s) and source are credited. 